\title{
Vanishing enstrophy dissipation in two-dimensional Navier-Stokes turbulence in the inviscid limit
}

\author{
By CHUONG V. TRAN AND DAVID G. DRITSCHEL \\ School of Mathematics and Statistics, University of St Andrews, St Andrews KY16 9SS, UK
}

(Received 12 December 2005 and in revised form 20 April 2006)

Batchelor (Phys. Fluids, vol. 12, 1969, p. 233) developed a theory of two-dimensional turbulence based on the assumption that the dissipation of enstrophy (mean-square vorticity) tends to a finite non-zero constant in the limit of infinite Reynolds number $R e$. Here, by assuming power-law spectra, including the one predicted by Batchelor's theory, we prove that the maximum dissipation of enstrophy is in fact zero in this limit. Specifically, as $R e \rightarrow \infty$, the dissipation approaches zero no slower than $(\ln R e)^{-1 / 2}$. The physical reason behind this result is that the decrease of viscosity enhances the production of both palinstrophy (mean-square vorticity gradients) and its dissipation - but in such a way that the net growth of palinstrophy is less rapid than the decrease of viscosity, resulting in vanishing enstrophy dissipation. This result generalizes to a rich class of quasi-geostrophic models as well as to the case of a passive tracer in layerwise-two-dimensional turbulent flows having bounded enstrophy.

\section{Introduction}

The evolution of an unforced two-dimensional incompressible fluid under the effects of molecular viscosity is governed by the vorticity equation,

$$
q_{t}+J(\psi, q)=v \Delta q,
$$

where $q(x, y, t)$ is the vorticity, $J(\phi, \varphi)=\phi_{x} \varphi_{y}-\phi_{y} \varphi_{x}, v$ is the kinematic viscosity and $\psi(x, y, t)$ is the streamfunction. The vorticity, streamfunction and fluid velocity $\boldsymbol{u}$ are related by $q=\Delta \psi=\hat{\boldsymbol{n}} \cdot \nabla \times \boldsymbol{u}$, or equivalently $\boldsymbol{u}=\left(-\psi_{y}, \psi_{x}\right)=\left(-\Delta^{-1} q_{y}, \Delta^{-1} q_{x}\right)$, where $\hat{\boldsymbol{n}}$ is the normal vector to the fluid domain. All fields are assumed to be periodic in both $x$ and $y$ and to have zero average.

Equation (1.1) has been actively studied for decades. An important question concerns the direct transfer of the mean-square vorticity (twice the enstrophy) and its dissipation for high Reynolds numbers. This problem is interesting as it resembles the direct transfer of energy and its dissipation in a three-dimensional fluid. In a seminal work on this subject, Batchelor (1969) predicted that when an initial vorticity reservoir spreads out in a virtually inviscid region of wavenumber space, an enstrophy spectrum proportional to $k^{-1}$ forms in the 'inertial range'. This uniform redistribution of enstrophy among the wavenumber octaves of the inertial range eventually reaches a scale around which viscous dissipation is considerable, resulting in a non-negligible enstrophy dissipation rate. In the inviscid limit, this dissipation rate is assumed to remain finite, i.e. the growth of the mean-square vorticity gradients (twice the palinstrophy) is assumed to be proportional to $v^{-1}$ as $v \rightarrow 0$. 
Batchelor's theory has been vigorously examined, mostly by numerical methods and qualitative analyses, and the research performed on this subject constitutes an extensive literature (see, for example, Bartello \& Warn 1996; Chasnov 1997; Bracco et al. 2000; Davidson 2004; Dmitruk \& Montgomery 2005). A recent result by Tran (2005) provides an upper bound for the enstrophy dissipation (see below) that depends only on the initial vorticity distribution. For the numerics, a major challenge is to resolve not only the vorticity gradients responsible for the enstrophy dissipation, but also the Laplacian $\Delta q$. This is because the palinstrophy dissipation (and possibly its production, see $\S 2$ ) is determined by $\Delta q$, so a poor resolution of $\Delta q$ will inevitably lead to spurious palinstrophy dynamics, thereby returning unreliable enstrophy dissipation rates. Nevertheless, serious attempts have been made with the available resolutions to explore the problem of enstrophy decay, and interesting predictions have been put forth. In particular, Dmitruk \& Montgomery (2005), on the basis of their extrapolation of simulated results for low Reynolds numbers to higher ones, suggest that the enstrophy dissipation may vanish in the inviscid limit.

In this paper we show that this is indeed the case. More precisely, we show that in the inviscid limit, the redistribution of vorticity from an initial reservoir via an enstrophy spectrum no shallower than the classical $k^{-1}$ spectrum results in vanishing enstrophy dissipation. Essentially, the decrease of viscosity enhances the production of both palinstrophy and its dissipation in such a way that the net growth of palinstrophy is less rapid than the decrease of viscosity, resulting in vanishing enstrophy dissipation. This result generalizes, for both an active scalar and a passive tracer, advected by layerwise-two-dimensional turbulent flows governed by a rich class of quasi-geostrophic models.

\section{Mathematical background}

\subsection{Basic identities}

By straightforward calculation we have

$$
\Delta J(\psi, q)=J(\psi, \Delta q)+2 J\left(\psi_{x}, q_{x}\right)+2 J\left(\psi_{y}, q_{y}\right) .
$$

Multiplying this by $q$ and taking the spatial average of the resulting equation yields

$$
\langle\Delta q J(\psi, q)\rangle=\langle q \Delta J(\psi, q)\rangle=\left\langle q J\left(\psi_{x}, q_{x}\right)\right\rangle+\left\langle q J\left(\psi_{y}, q_{y}\right)\right\rangle,
$$

where the identity $\langle q J(\psi, \Delta q)\rangle=-\langle\Delta q J(\psi, q)\rangle$ has been used.

\subsection{Decaying Casimirs}

In the absence of the viscous term, (1.1) expresses material conservation of $q$. This gives rise to an infinite class of conserved quantities. In general, the average value $\langle f(q)\rangle$ of an arbitrary twice differentiable function $f(q)$ is conserved. These invariants are known as Casimirs, and we shall adopt this terminology below.

Under viscous effects, a wide class of these Casimirs decays in time. Here the term 'decays' is used in a liberal sense: a positive Casimir decays if it suffers a negative growth rate, regardless of the functional form of the resulting viscous term. In general, if $f(q)>0$ is convex, i.e. $f^{\prime \prime}(q)>0$, then $\langle f(q)\rangle$ decays. This can be seen from the evolution equation of $\langle f(q)\rangle$,

$$
\begin{aligned}
\frac{\mathrm{d}}{\mathrm{d} t}\langle f(q)\rangle=\left\langle f^{\prime}(q) q_{t}\right\rangle & =-\left\langle f^{\prime}(q) J(\psi, q)\right\rangle+v\left\langle f^{\prime}(q) \Delta q\right\rangle \\
& =\left\langle\psi f^{\prime \prime}(q) J(q, q)\right\rangle-v\left\langle f^{\prime \prime}(q)|\nabla q|^{2}\right\rangle \\
& =-v\left\langle f^{\prime \prime}(q)|\nabla q|^{2}\right\rangle
\end{aligned}
$$


where the right-hand side follows from integration by parts, upon which the tripleproduct term identically vanishes. Therefore, $\mathrm{d}\langle f(q)\rangle / \mathrm{d} t<0$ if $f^{\prime \prime}(q)>0$, implying a decay of $\langle f(q)\rangle$. This result applies to the case $f(q)=|q|^{p}$ for $p>1$. For this case, the following slightly varied version of (2.3),

$$
\frac{\mathrm{d}}{\mathrm{d} t}\left\langle|q|^{p}\right\rangle^{1 / p}=-v(p-1)\left\langle|q|^{p}\right\rangle^{1 / p-1}\left\langle|q|^{p-2}|\nabla q|^{2}\right\rangle,
$$

gives explicit decay rates for the $L^{p}$ norms $\left\langle|q|^{p}\right\rangle^{1 / p}$. The most important decaying quantities in this subclass are the $L^{2}$ norm $\left\langle q^{2}\right\rangle^{1 / 2}$ and $L^{\infty}$ norm $\|q\|_{\infty}$, the latter being obtained by taking $\left\langle|q|^{p}\right\rangle^{1 / p}$ to the limit $p \rightarrow \infty$. The $L^{\infty}$ norm appears to be better conserved in practice than the $L^{2}$ norm, and may even be better conserved than the energy. These two fundamental dynamical quantities appear repeatedly in subsequent calculations.

\subsection{An upper bound for the enstrophy dissipation rate $v\left\langle|\nabla q|^{2}\right\rangle$}

The nonlinear transfer of vorticity to smaller scales necessarily results in a rapid growth of vorticity gradients, and this growth is enhanced as $v$ is decreased. However, this growth of vorticity gradients must be accompanied by ever greater viscous effects that work against such growth. The competition between vorticity gradient production and viscous dissipation holds the key to understanding the present problem. The outcome of this competition, according to Batchelor (1969), is that the enstrophy dissipation rate $v\left\langle|\nabla q|^{2}\right\rangle$ remains finite in the limit $v \rightarrow 0$. This means that $\left\langle|\nabla q|^{2}\right\rangle$ diverges as $v^{-1}$ in that limit. Recently, Tran (2005) has shown that, for so long as the rate of enstrophy dissipation is increasing, it remains bounded above by a quantity that depends only on the initial vorticity distribution: $v\left\langle|\nabla q|^{2}\right\rangle \leqslant\|q(0)\|_{\infty}\left\langle q^{2}(0)\right\rangle$. This upper bound applies during palinstrophy growth, including the palinstrophy peak. This result is consistent with Batchelor's prediction, but does not rule out the possibility $v\left\langle|\nabla q|^{2}\right\rangle \rightarrow 0$ in the limit $v \rightarrow 0$. In fact, further analyses based on this result, carried out below, suggest the realizability of this possibility.

We now re-derive the aforementioned upper bound, which is the basis for subsequent analyses. By multiplying (1.1) by $\Delta q$ and taking the spatial average of the resulting equation, we obtain the equation governing the evolution of the palinstrophy $\left\langle|\nabla q|^{2}\right\rangle / 2$,

$$
\begin{aligned}
\frac{1}{2} \frac{\mathrm{d}}{\mathrm{d} t}\left\langle|\nabla q|^{2}\right\rangle & =\langle\Delta q J(\psi, q)\rangle-v\left\langle|\Delta q|^{2}\right\rangle \\
& =\left\langle q J\left(\psi_{x}, q_{x}\right)\right\rangle+\left\langle q J\left(\psi_{y}, q_{y}\right)\right\rangle-v\left\langle|\Delta q|^{2}\right\rangle \\
& \leqslant\left\langle|q|\left(\left|\nabla \psi_{x}\right|\left|\nabla q_{x}\right|+\left|\nabla \psi_{y}\right|\left|\nabla q_{y}\right|\right)\right\rangle-v\left\langle|\Delta q|^{2}\right\rangle \\
& \leqslant\|q\|_{\infty}\left\langle\left|\nabla \psi_{x}\right|^{2}+\left|\nabla \psi_{y}\right|^{2}\right\rangle^{1 / 2}\left\langle\left|\nabla q_{x}\right|^{2}+\left|\nabla q_{y}\right|^{2}\right\rangle^{1 / 2}-v\left\langle|\Delta q|^{2}\right\rangle \\
& =\|q\|_{\infty}\left\langle q^{2}\right\rangle^{1 / 2}\left\langle|\Delta q|^{2}\right\rangle^{1 / 2}-v\left\langle|\Delta q|^{2}\right\rangle \\
& =\left\langle|\Delta q|^{2}\right\rangle^{1 / 2}\left(\|q\|_{\infty}\left\langle q^{2}\right\rangle^{1 / 2}-v\left\langle|\Delta q|^{2}\right\rangle^{1 / 2}\right) .
\end{aligned}
$$

The triple-product term $\|q\|_{\infty}\left\langle q^{2}\right\rangle^{1 / 2}\left\langle|\Delta q|^{2}\right\rangle^{1 / 2}$ represents an upper bound for the production rate of $\left\langle|\nabla q|^{2}\right\rangle / 2$. In (2.5), the second equation is obtained via the identity (2.2) and all other steps are straightforward. This equation is the basis for the analyses in the next section. Applying the Cauchy-Schwarz inequality $\left\langle|\nabla q|^{2}\right\rangle \leqslant$ $\left\langle|\Delta q|^{2}\right\rangle^{1 / 2}\left\langle q^{2}\right\rangle^{1 / 2}$ to $(2.5)$ yields

$$
\frac{1}{2} \frac{\mathrm{d}}{\mathrm{d} t}\left\langle|\nabla q|^{2}\right\rangle \leqslant\left(\frac{\left\langle|\Delta q|^{2}\right\rangle}{\left\langle q^{2}\right\rangle}\right)^{1 / 2}\left(\|q\|_{\infty}\left\langle q^{2}\right\rangle-v\left\langle|\nabla q|^{2}\right\rangle\right) .
$$


Since both $\|q\|_{\infty}$ and $\left\langle q^{2}\right\rangle$ decay with time, it follows that if $v\left\langle|\nabla q|^{2}\right\rangle \leqslant\|q\|_{\infty}\left\langle q^{2}\right\rangle$ holds for $t=0$, then

$$
v\left\langle|\nabla q|^{2}\right\rangle \leqslant\|q(0)\|_{\infty}\left\langle q^{2}(0)\right\rangle \text { for } t>0
$$

\section{Enstrophy dissipation in the limit $v \rightarrow 0$}

This section contains further analyses of (2.5) by a semi-analytic method. The essential idea is to exploit the delicate balance between the production and dissipation of palinstrophy. This balance has been lost in (2.6) after the employment of the Cauchy-Schwarz inequality, which can be excessively generous in the present context, effectively giving an edge to the palinstrophy production term. One can see that this inequality is sharp when $q$ is spectrally supported by a narrow band of wavenumbers (becoming an equality only when the support consists of a single wavenumber) and becomes increasingly excessive when the support becomes increasingly broader. Now the growth of $\left\langle|\nabla q|^{2}\right\rangle$ from a band-limited initial reservoir by direct enstrophy transfer is precisely the mechanism for the Cauchy-Schwarz inequality to become excessive. By sacrificing a reasonable degree of rigour, we derive estimates that are sharper than (2.6), allowing us to deduce that the enstrophy dissipation vanishes in the inviscid limit. This result means that the transition from viscous to inviscid dynamics is 'smooth' and helps to resolve the fundamental and long-standing question of whether two-dimensional turbulence at high Reynolds numbers can be meaningfully described by the two-dimensional Euler equations.

\subsection{Preliminaries}

For the remainder of this paper, approximate power-law enstrophy spectra will be assumed for the enstrophy inertial range, followed by a steeper dissipation range. This assumption is customary in the study of turbulence and seems reasonable for a high-Reynolds-number inertial range whose spectrum represents an average over a vast number of wavenumbers. This assumption is even more plausible within a statistical framework, which can be formulated in a straightforward manner for the present problem. Consider an ensemble of vorticity reservoirs, possibly with quite different levels of bounded energy and enstrophy. Let us denote by $\bar{X}$ the average, taken over the ensemble, of a dynamical quantity $X$. From the penultimate line in (2.5) we can deduce that

$$
\frac{1}{2} \frac{\mathrm{d}}{\mathrm{d} t} \overline{\left\langle|\nabla q|^{2}\right\rangle} \leqslant{\overline{\left\langle|\Delta q|^{2}\right\rangle}}^{1 / 2}\left({\overline{\left(\mid q \|_{\infty}^{2}\left\langle q^{2}\right\rangle\right.}}^{1 / 2}-v{\overline{\left\langle|\Delta q|^{2}\right\rangle}}^{1 / 2}\right) .
$$

This has the same form as (2.5), apart from minor differences coming from the ensemble mean over the product $\|q\|_{\infty}^{2}\left\langle q^{2}\right\rangle$.

Henceforth, we consider (2.5) without loss of generality. Since the first term in the brackets on the right-hand side of the final line of (2.5) is bounded from above in terms of initial data and independent of the Reynolds number, the net growth of palinstrophy in the limit $v \rightarrow 0$ may not be so dramatic and depends critically on how $\left\langle|\Delta q|^{2}\right\rangle$ responds to that limit. In order to explore the outcome of the competition between the palinstrophy production and dissipation, we rewrite (2.5) in the form

$$
\frac{1}{2} \frac{\mathrm{d}}{\mathrm{d} t}\left\langle|\nabla q|^{2}\right\rangle \leqslant \frac{\left\langle|\Delta q|^{2}\right\rangle}{\left\langle|\nabla q|^{2}\right\rangle}\left(\|q\|_{\infty}\left\langle q^{2}\right\rangle^{1 / 2} \frac{\left\langle|\nabla q|^{2}\right\rangle}{\left\langle|\Delta q|^{2}\right\rangle^{1 / 2}}-v\left\langle|\nabla q|^{2}\right\rangle\right) .
$$

During the period (or periods) of palinstrophy growth, the enstrophy dissipation is bounded from above by the first term in the brackets. The global maximum of the 
enstrophy dissipation is also bounded in the same manner, i.e.

$$
v\left\langle|\nabla q|^{2}\right\rangle \leqslant\|q\|_{\infty}\left\langle q^{2}\right\rangle^{1 / 2} \frac{\left\langle|\nabla q|^{2}\right\rangle}{\left\langle|\Delta q|^{2}\right\rangle^{1 / 2}} .
$$

Suppose that $v\left\langle|\nabla q|^{2}\right\rangle \rightarrow \chi>0$ as $v \rightarrow 0$, then $\chi$ is also a lower bound for the term on the right-hand side of (3.3). But it is quite easy to find examples of power-law spectra such that both the palinstrophy diverges and the right-hand side of (3.3) vanishes. Such spectra are therefore incompatible with a finite enstrophy dissipation. This means that these spectra could be achieved only in the presence of vanishing enstrophy dissipation. This argument turns out to cover virtually all spectra of interest, which are examined in what follows.

We allow for mean-square vorticity spectra of the form

$$
Q(k)= \begin{cases}C k^{-\beta} & \text { if } k_{0} \leqslant k \leqslant k_{v}, \\ D(k) & \text { if } k \geqslant k_{v}\end{cases}
$$

where the power-law scaling $C k^{-\beta}$ is assumed for the inertial range $\left[k_{0}, k_{\nu}\right]$, followed by a steeper spectrum $D(k)$ in the dissipation range. We further assume a divergence of $k_{v}$ in the inviscid limit $\uparrow$ and that

$$
C \int_{k_{0}}^{k_{v}} k^{2-\beta} \mathrm{d} k=c^{-1} \int_{k_{v}}^{\infty} k^{2} D(k) \mathrm{d} k,
$$

where $c$ is a constant of order unity. This means that in the inviscid limit the inertial range becomes infinitely wide and that the enstrophy is most strongly dissipated around $k_{v}$ or beyond. For $c=1$, (3.5) states that as much enstrophy dissipation is occurring for $k>k_{v}$ as for $k<k_{v}$. We could indeed define $k_{v}$ by the condition $c=1$.

We pause to remark that the true spectrum may fluctuate about the ideal power law assumed, even for an ensemble of vorticity reservoirs. This is permissible in the present analysis so long as the fluctuations are bounded by the ideal spectrum as $k$ tends to $k_{v}$, specifically over a sufficiently broad range of wavenumbers contributing to the integrated spectra. In other words, our principle assumption is that the fluctuations contribute less to the integrated spectra than do the ideal power-law parts. This appears reasonable on statistical grounds, since the sample size of wavenumbers in a shell of constant $k$ increases like $k$ itself, implying that fluctuations diminish (assuming approximate isotropy) relative to the ideal spectrum. Numerical results at very high Reynolds numbers support this assumption, see Dmitruk \& Montgomery (2005). Here, we can even tolerate fluctuations which remain comparable to but marginally less than the ideal power-law spectra considered.

A range of values for $\beta$ exhausting all possibilities is considered. Since we are interested in the limit $\left\langle|\nabla q|^{2}\right\rangle \rightarrow \infty$, the case $\beta>3$ can be excluded a priori since this case corresponds to $\left\langle|\nabla q|^{2}\right\rangle<\infty$ because its spectrum $k^{2} Q(k)$ is steeper than $k^{-1}$. On physical grounds, we also ignore the case $\beta<1$ since this would violate the dual conservation of energy and enstrophy. The reason is that the assumed inertial-range enstrophy spectrum, when shallower than $k^{-1}$, either has a given amount of energy (around $k_{0}$ ) and an arbitrarily large amount of enstrophy (around $k_{v} \rightarrow \infty$ ) or has a given amount of enstrophy (around $k_{v} \rightarrow \infty$ ) and an arbitrarily small amount of energy (around $k_{0}$ ). Hence a given reservoir of finite energy and enstrophy cannot

$\dagger$ The manner in which $k_{v}$ diverges and details of the spectrum in the dissipation range are interesting, but immaterial for the present arguments. 
possibly relax into such a spectrum. More rigorously, the characteristic wavenumber $s \equiv\left(\left\langle|q|^{2}\right\rangle /\left\langle|\nabla \psi|^{2}\right\rangle\right)^{1 / 2}$ can only decay in time (Tran \& Shepherd 2002), which forbids the enstrophy spectrum becoming shallower than $k^{-1}$.

Now the redistribution of enstrophy via a spectrum shallower than $k^{-1}$ over a limited wavenumber interval $\left[k_{0}, k_{1}\right]$, followed by a range $\left[k_{1}, k_{v}\right]$ steeper than $k^{-1}$ (plus a still steeper dissipation range) cannot be logically excluded. However, we will see that this case can be treated in the same way as the assumed case (3.4) with $1 \leqslant \beta \leqslant 3$ since the contributions by $k \in\left[k_{0}, k_{1}\right]$ to the palinstrophy and to its dissipation are negligible compared with those by $k>k_{1}$ in the limit $k_{v} \rightarrow \infty$. In summary, we can restrict our consideration to the assumed spectrum (3.4) with $1 \leqslant \beta \leqslant 3$. Finally, if one abandons the usual notion of power-law scaling, then the picture of an initial reservoir evolving into an extremely polarized spectrum with energy residing at the lower-wavenumber end and enstrophy residing at the higher-wavenumber end (with virtually nothing in between) cannot be ruled out. The implication of this hypothetical picture for the enstrophy dissipation in the inviscid limit will be discussed at the end of this section, after exhausting other possibilities.

\subsection{The case $\beta>1$}

By definition we have

$$
\left\langle q^{2}\right\rangle=\int Q(k) \mathrm{d} k=C \int k^{-\beta} \mathrm{d} k=\frac{C}{\beta-1} k_{0}^{1-\beta}
$$

(valid for $k_{v} \rightarrow \infty$ ). It follows that $C=(\beta-1) k_{0}^{\beta-1}\left\langle q^{2}\right\rangle$. For $\beta<3$, the quantities $\left\langle|\nabla q|^{2}\right\rangle$ and $\left\langle|\Delta q|^{2}\right\rangle^{1 / 2}$ are estimated as follows:

$$
\left\langle|\nabla q|^{2}\right\rangle=\int k^{2} Q(k) \mathrm{d} k=C \int_{k_{0}}^{k_{v}} k^{2-\beta} \mathrm{d} k+\int_{k_{v}}^{\infty} k^{2} D(k) \mathrm{d} k=C \frac{1+c}{3-\beta} k_{v}^{3-\beta}
$$

and

$$
\begin{aligned}
\left\langle|\Delta q|^{2}\right\rangle^{1 / 2} & =\left(\int k^{4} Q(k) \mathrm{d} k\right)^{1 / 2}=\left(C \int_{k_{0}}^{k_{v}} k^{4-\beta} \mathrm{d} k+\int_{k_{v}}^{\infty} k^{4} D(k) \mathrm{d} k\right)^{1 / 2} \\
& \geqslant\left(C \int_{k_{0}}^{k_{v}} k^{4-\beta} \mathrm{d} k+k_{v}^{2} \int_{k_{v}}^{\infty} k^{2} D(k) \mathrm{d} k\right)^{1 / 2}=\left(\frac{C}{5-\beta} k_{v}^{5-\beta}+\frac{C c}{3-\beta} k_{v}^{5-\beta}\right)^{1 / 2} \\
& =C^{1 / 2}\left(\frac{1}{5-\beta}+\frac{c}{3-\beta}\right)^{1 / 2} k_{v}^{3-\beta} k_{v}^{(\beta-1) / 2}
\end{aligned}
$$

With (3.6) and (3.7) the upper bound (3.3) becomes

$$
v\left\langle|\nabla q|^{2}\right\rangle \leqslant C^{1 / 2} k_{v}^{(1-\beta) / 2}\|q\|_{\infty}\left\langle q^{2}\right\rangle^{1 / 2},
$$

where a dimensionless factor of order unity has been dropped. For $\beta>1$ the righthand side of (3.8) vanishes as $k_{v} \rightarrow \infty$. Hence, in the limit $v \rightarrow 0$, if the turbulence evolves while maintaining this spectral slope and if $k_{v} \rightarrow \infty$, then the enstrophy dissipation vanishes in that limit. (Note that a finite $k_{v}$ would correspond to a finite palinstrophy, which would further imply vanishing enstrophy dissipation.)

For the marginal case $\beta=3$, we have $\left\langle|\nabla q|^{2}\right\rangle \approx C \ln \left(k_{v} / k_{0}\right)$ and $\left\langle|\Delta q|^{2}\right\rangle^{1 / 2} \approx C^{1 / 2} k_{\nu}$, and the arguments of vanishing enstrophy dissipation in the preceding paragraph apply without change. 


\subsection{The classical case $\beta=1$}

There remains the critical case $\beta=1$, which is the classical prediction. For this case, $C$ is given by $C=\left\langle q^{2}\right\rangle / \ln \left(k_{v} / k_{0}\right)$ (ignoring the contribution to the enstrophy from $D(k)$, which would result in a constant of order unity in the denominator), and the two estimates (3.6) and (3.7) become

$$
\left\langle|\nabla q|^{2}\right\rangle=\frac{(1+c)\left\langle q^{2}\right\rangle}{2 \ln \left(k_{v} / k_{0}\right)} k_{v}^{2}
$$

and

$$
\left\langle|\Delta q|^{2}\right\rangle^{1 / 2} \geqslant \frac{(1+2 c)^{1 / 2}\left\langle q^{2}\right\rangle^{1 / 2}}{2\left[\ln \left(k_{v} / k_{0}\right)\right]^{1 / 2}} k_{v}^{2} .
$$

The upper bound (3.3) takes the form

$$
v\left\langle|\nabla q|^{2}\right\rangle \leqslant \frac{\|q\|_{\infty}\left\langle q^{2}\right\rangle}{\left[\ln \left(k_{v} / k_{0}\right)\right]^{1 / 2}},
$$

where a dimensionless factor of order unity has been dropped. Since $\ln \left(k_{\nu} / k_{0}\right)$ diverges as $v \rightarrow 0$, (3.11) implies vanishing enstrophy dissipation as before. Note that $v\left\langle|\nabla q|^{2}\right\rangle$ may approach zero, as $v \rightarrow 0$, far more slowly than in the previous case.

It can be seen that the conclusion of vanishing enstrophy dissipation applies to the case of the log-corrected spectrum considered by Kraichnan (1971). One may consider this spectrum as marginally steeper than $k^{-1}$, which the preceding and present subsections have covered.

The upper bound (3.11) readily allows one to deduce how the enstrophy dissipation decreases with viscosity. We first estimate the ratio $k_{v} / k_{0}$ in terms of known quantities. By (2.5), at the palinstrophy peak we have

$$
v\left\langle|\Delta q|^{2}\right\rangle^{1 / 2} \leqslant\|q\|_{\infty}\left\langle q^{2}\right\rangle^{1 / 2}
$$

Substituting (3.12) into (3.10) and solving for $k_{v}$, neglecting the constant factor of order unity, yields

$$
k_{v} \leqslant \frac{\|q\|_{\infty}^{1 / 2}\left[\ln \left(k_{v} / k_{0}\right)\right]^{1 / 4}}{v^{1 / 2}} .
$$

We now replace $k_{0}$ by the enstrophy centroid wavenumber $s=\left\langle q^{2}\right\rangle^{1 / 2} /\left\langle|\nabla \psi|^{2}\right\rangle^{1 / 2}$. This is equivalent to saying that most of the enstrophy is directly transferred from $s$ to the higher wavenumbers. The ratio $k_{v} / k_{0}$ can then be estimated by

$$
\frac{k_{v}}{k_{0}} \approx \frac{\|q\|_{\infty}^{1 / 2}}{v^{1 / 2} s} .
$$

(Preliminary calculations indicate that this estimate for $k_{v}$ is comparable to that found from setting $c=1$ in (3.5).) Substituting this ratio into (3.11) yields

$$
v\left\langle|\nabla q|^{2}\right\rangle \leqslant \frac{\|q\|_{\infty}\left\langle q^{2}\right\rangle}{(\ln R e)^{1 / 2}},
$$

where the Reynolds number $R e$ takes the form

$$
\operatorname{Re}=\frac{\|q\|_{\infty}}{\nu s^{2}}
$$


This result qualitatively describes the dependence of the enstrophy dissipation on $R e$. In the limit $R e \rightarrow \infty$, the enstrophy dissipation approaches zero no slower than $(\ln R e)^{-1 / 2}$. Incidentally, Dmitruk \& Montgomery (2005) suggest the dependence $(\ln R e)^{-1}$ of the enstrophy dissipation on the Reynolds number.

Finally, for the sake of completeness, the hypothetical state mentioned in $\S 3.1$ of an energy-enstrophy 'dipole' developed from an initial reservoir is examined. Let us now denote by $k_{v}$ the wavenumber around which the enstrophy 'monopole' resides. In this case we have $\left\langle|\nabla q|^{2}\right\rangle \approx\left\langle q^{2}\right\rangle k_{v}^{2}$ and $\left\langle|\Delta q|^{2}\right\rangle \approx\left\langle q^{2}\right\rangle k_{v}^{4}$, and the above arguments of vanishing enstrophy dissipation would fail. Note that this state requires not only the enstrophy but also both the palinstrophy and its dissipation to be sharply confined around $k_{v}$. We know of no theoretical or numerical evidence for the persistence of such a state.

\section{Generalization to quasi-geostrophic flows}

We now allow $q$ to be a general scalar, either an active scalar or a passive tracer, advected by layerwise-two-dimensional turbulent flows governed by a rich class of quasi-geostrophic models. In what follows we discuss several cases, to which the results in $\S 2$ and $\S 3$ can be readily extended.

The case $q=\Delta \psi(x, y, z, t)$ corresponds to a quasi-geostrophic model, which is derived under the assumption that both the buoyancy and rotation frequencies are constant and with the vertical coordinate scaled by their ratio (see Gill 1982, p. 530; Reinaud, Dritschel \& Koudella 2003). Periodic boundary conditions are conveniently extended to the vertical dimension and the Laplacian is accordingly extended. This means that $q$ (known as the potential vorticity) is horizontally advected by the twodimensional incompressible velocity field $\left(-\psi_{y}, \psi_{x}\right)$, but homogeneously diffused in three-space. The $z$-dependence of $q$ and $\psi$ introduces the additional term $\left\langle q J\left(\psi_{z}, q_{z}\right)\right\rangle$ to the right-hand side of (2.2), requiring (2.5) to be modified accordingly. However, such minor modifications do not alter the final results and conclusions. More precisely, the constraint (2.7) and all arguments in $\S 3$ remain unaltered.

A different form of the potential vorticity, $q=\left(\Delta-\lambda^{2}\right) \psi(x, y, t)$, arises from a popular quasi-geostrophic shallow-water model known as the Charney-HasegawaMima model (see Tran \& Dritschel 2006 and references therein). In this case, the enstrophy is not conserved but bounded from above by $\left\langle|\Delta \psi|^{2}\right\rangle \leqslant\langle\Delta \psi q\rangle \leqslant\left\langle q^{2}\right\rangle$, where $\langle\Delta \psi q\rangle / 2$ is known as the potential enstrophy, which is not a decaying Casimir but nevertheless a decaying quadratic. With this upper bound, the constraint (2.7) and the arguments in $\S 3$ remain intact. For sharper estimates one can replace $\left\langle q^{2}\right\rangle$ by $\langle\Delta \psi q\rangle$ in (2.5), and by doing that the constraint (2.7) becomes

$$
v\left\langle|\nabla q|^{2}\right\rangle \leqslant\|q(0)\|_{\infty}\langle\Delta \psi(0) q(0)\rangle^{1 / 2}\left\langle q^{2}(0)\right\rangle^{1 / 2} .
$$

The active scalar $q=(-\Delta)^{\alpha / 2} \psi(x, y, t)$, for $\alpha>0$, was first considered by Pierrehumbert, Held \& Swanson (1994) as a generalized version of the usual vorticity $\Delta \psi$. Besides this popular case, there are two other physically relevant cases, $q=(-\Delta)^{1 / 2} \psi$ and $q=(-\Delta)^{3 / 2} \psi$, the former of which has been widely studied (see Tran 2006 and references therein). The scalar $q=(-\Delta)^{1 / 2} \psi$, corresponding to $\alpha=1$, represents the surface temperature in the so-called surface quasi-geostrophic model. The scalar $q=(-\Delta)^{3 / 2} \psi$, corresponding to $\alpha=3$, appears in the equation governing a shallow flow in a rotating domain with uniform internal heating. For general $\alpha>0$, the advective term conserves the two quadratic quantities $\left\langle\left|(-\Delta)^{\alpha / 4} \psi\right|^{2}\right\rangle$ and $\left\langle q^{2}\right\rangle=\left\langle\left|(-\Delta)^{\alpha / 2} \psi\right|^{2}\right\rangle$, both of which decay under diffusive effects. The enstrophy is one 
of the invariants for $\alpha=2,4$, the former being the two-dimensional Navier-Stokes case. For $\alpha=4$, the constraint (2.7) becomes

$$
v\left\langle|\nabla q|^{2}\right\rangle \leqslant\|q(0)\|_{\infty}\left\langle|\Delta \psi(0)|^{2}\right\rangle^{1 / 2}\left\langle q^{2}(0)\right\rangle^{1 / 2},
$$

and the arguments of vanishing diffusion remain valid. For $\alpha \in(2,4)$, the enstrophy is no longer conserved by nonlinear transfer. However, it can be bounded from above in terms of the two invariants. Namely, we have $\left\langle|\Delta \psi|^{2}\right\rangle \leqslant\left\langle\left|(-\Delta)^{\alpha / 4} \psi\right|^{2}\right\rangle^{2-4 / \alpha}\left\langle q^{2}\right\rangle^{4 / \alpha-1}$ (see Tran 2004). With this upper bound, the constraint (2.7) takes the form

$$
v\left\langle|\nabla q|^{2}\right\rangle \leqslant\|q(0)\|_{\infty}\left\langle\left|(-\Delta)^{\alpha / 4} \psi(0)\right|^{2}\right\rangle^{1-2 / \alpha}\left\langle q^{2}(0)\right\rangle^{2 / \alpha} .
$$

Again the arguments of vanishing diffusion remain valid. When $\alpha \notin[2,4]$, enstrophy is expected to be generated by nonlinear transfer (see Tran 2004). This jeopardizes the enstrophy boundedness as $v \rightarrow 0$, and the present results cannot be guaranteed to hold without further consideration.

Finally, for a passive tracer $q$ advected by two-dimensional flows having bounded enstrophy - but otherwise either forced-dissipative or decaying or unforced and inviscid - all the results in $\S 2$ and $\S 3$ apply with minor modifications. In particular, (2.7) becomes

$$
v\left\langle|\nabla q|^{2}\right\rangle \leqslant\|q(0)\|_{\infty}\left\langle|\Delta \psi|^{2}\right\rangle_{M}^{1 / 2}\left\langle q^{2}(0)\right\rangle^{1 / 2}
$$

where $\left\langle|\Delta \psi|^{2}\right\rangle_{M}$ denotes the maximum over time of the fluid mean-square vorticity.

\section{Concluding remarks}

The nature of enstrophy dissipation in the inviscid limit of the two-dimensional Navier-Stokes equations has been a long-standing problem in classical fluid mechanics. Batchelor (1969) assumed a finite enstrophy dissipation, and used scaling arguments to predict a $k^{-1}$ form of the enstrophy spectrum in the inertial range. Here, we have shown that the balance between palinstrophy production and dissipation is delicate: further constraints not used by Batchelor (1969) indicate that the growth of palinstrophy (mean-square vorticity gradients) as the viscosity $v \rightarrow 0$ is marginally less rapid than the decrease of viscosity. This is because the nonlinear term responsible for the production of palinstrophy is proportional to $\left\langle|\Delta q|^{2}\right\rangle^{1 / 2}$, which is relatively weak compared to the viscous dissipation term $v\left\langle|\Delta q|^{2}\right\rangle$. Ultimately, as $v \rightarrow 0$, viscous dissipation of palinstrophy remains able to counter-balance the nonlinear production of palinstrophy, resulting in vanishing enstrophy dissipation. For Batchelor's spectrum, the dissipation is bounded by $(\ln R e)^{-1 / 2}$ as the Reynolds number $R e \rightarrow \infty$, an extraordinarily weak dependence. But for any steeper spectrum, the dissipation vanishes much more rapidly. This applies to a number of theories that do not rely on the assumption of finite enstrophy dissipation and suggest spectra steeper than Batchelor's spectrum (see Saffman 1971; Sulem \& Frisch 1975; Moffatt 1987). In short, there may be no link between enstrophy dissipation in two-dimensional turbulence and energy dissipation in three-dimensional turbulence as envisioned by Batchelor (1969).

Batchelor (1969) used the expected finite value of enstrophy dissipation in his scaling argument to arrive at the $k^{-1}$ enstrophy spectrum. Perhaps Batchelor's argument is not so sensitive to the extraordinarily weak dependence of enstrophy dissipation on Reynolds number that we have found here. In fact if we replace the limiting value of the enstrophy dissipation by the non-zero maximum enstrophy dissipation achieved for a particular $R e$, Batchelor's scaling analysis may still apply. 
Vanishing dissipation of scalar variance occurs in a wide variety of geophysical fluid models, including the Charney-Hasegawa-Mima model describing a shallowwater fluid with a free surface, the $\alpha$ turbulence model for $2 \leqslant \alpha \leqslant 4$, and the three-dimensional quasi-geostrophic model with constant buoyancy and rotation frequencies. The scalar may be active (as in the case of potential vorticity) or passive. In the latter case, the background flow need only have bounded mean-square vorticity.

We are grateful for the feedback received from three anonymous referees.

Note added in proof: While the paper was in press we became aware of an independent mathematical demonstration of vanishing enstrophy dissipation by Lopes, Mazzucato \& Lopes (2006), following on from Eyink (2001) and Di Perna \& Lions (1989).

\section{REFERENCES}

Bartello, P. \& Warn, T. 1996 Self-similarity of decaying two-dimensional turbulence. J. Fluid Mech. 326, 357-372.

BAtChelor, G. K. 1969 Computation of the energy spectrum in homogeneous two-dimensional turbulence. Phys. Fluids 12 Suppl. II, 233-239.

Bracco, A., McWilliams, J. C., Murante, G., Provenzale, A. \& Weiss, J. B. 2000 Revisiting freely decaying two-dimensional turbulence at millennial resolution. Phys. Fluids 12, 2931-2941.

Chasnov, J. R. 1997 On the decay of two-dimensional homogeneous turbulence. Phys. Fluids 9, $171-180$.

Davidson, P. A. 2004 Turbulence: An Introduction for Scientists and Engineers. Oxford University Press.

Di Perna, R. J. \& Lions, P. L. 1989 Ordinary differential equations, transport theory and Sobolev spaces. Invent. Maths 98, 511-547.

Dmitruk, P. \& Montgomery, D. C. 2005 Numerical study of the decay of enstrophy in a twodimensional Navier-Stokes fluid in the limit of very small viscosities. Phys. Fluids 17, 035114.

EYINK, G. L. 2001 Dissipation in turbulent solutions of 2D Euler equations. Nonlinearity 14, 787-802.

Gill, A. E. 1982 Atmosphere-Ocean Dynamics. Academic

Kraichnan, R. H. 1971 Inertial-range transfer in two- and three-dimensional turbulence. J. Fluid Mech. 47, 525-535.

Lopes, M. C., Mazzucato, A. L. \& Lopes, H. J. N. 2006 Weak solutions, renormalized solutions and enstrophy defects in 2D turbulence. Arch. Rat. Mech. Anal. 179, 353-387.

Moffatt, H. K. 1987 On Geophysical and astrophysical turbulence. In Advances in Turbulence (ed. G. Comte-Bellot \& J. Mathieu), pp. 228-244. Springer.

Pierrehumbert, R. T., Held, I. M., Swanson, K. L. 1994 Spectra of local and nonlocal twodimensional turbulence. Chaos Solitons Fract. 4, 1111-1116.

Reinaud, J. N., Dritschel, D. G. \& Koudella, C. R. 2003 The shape of vortices in quasigeostrophic turbulence. J. Fluid Mech. 474, 175-192.

SAFFMAN, P. G. 1971 Spectrum and decay of random 2-dimensional vorticity distributions at large Reynolds-number. Stud. Appl. Maths 50, 377-383.

Sulem, P. L. \& Frisch, U. 1975 Bounds on energy flux for finite-energy turbulence. J. Fluid Mech. 72, 417-423.

Tran, C. V. 2004 Nonlinear transfer and spectral distribution of energy in $\alpha$ turbulence. Physica D 191, 137-155.

Tran, C. V. 2005 Enstrophy dissipation in freely evolving two-dimensional turbulence. Phys. Fluids 17, 081704.

Tran, C. V. 2006 Diminishing inverse transfer and non-cascading dynamics in surface quasigeostrophic turbulence. Physica D 213, 76-84.

Tran, C. V. \& Dritschel, D. G. 2006 Impeded inverse energy transfer in the Charney-HasegawaMima model of quasi-geostrophic flows. J. Fluid Mech. 551, 435-443.

Tran, C. V. \& Shepherd, T. G. 2002 Constraints on the spectral distribution of energy and enstrophy dissipation in forced two-dimensional turbulence. Physica D 165, 199-212. 